〈制导与对抗〉

\title{
先进红外传感器对隐身飞机作用距离估算研究
}

\author{
何 苹 ${ }^{1}$, 王莹莹 ${ }^{2}$, 岳韶华 ${ }^{2}$
}

(1. 西京学院 信息工程学院, 陕西 西安 710123; 2. 空军工程大学 防空反导学院, 陕西 西安 710046)

\begin{abstract}
摘要: 为了有效地估算先进红外传感器对隐身飞机的作用距离, 以 F-22 与 F-35 进行对抗性仿真为想 定, 计算了 F-22 在长波和中波波段的红外辐射强度, 拟合了典型条件下大气透过率的经验公式, 推 测了 F-35 机载 IRST 和 AIM-9X 红外导引头的特征参数, 利用逐步逼近法, 计算了 F-35 机载 IRST 和 AIM-9X 导弹导引头对 F-22 的作用距离。计算结果表明, F-22 具有较强的红外隐身能力, 可使 F-35 的 IRST 对其迎头作用距离不大于 $62 \mathrm{~km}$, 但是 F-22 在近距作战中, 仍将受到红外格斗弹的较大威胁。 鉴于 F-22 的红外辐射随红外传感器探测角度的变化而敏感性高的特点, 建议飞机编队进行红外反隐 身探测时, 采用稀疏编队方式, 以提高探测概率。
\end{abstract}

关键词：隐身飞机；作用距离；IRST；红外格斗弹；F-22; F-35

中图分类号：TN219 文献标识码：A 文章编号：1001-8891(2020)06-0899-06

\section{Operating Range of the Advanced Infrared Detector for the Stealth Aircraft}

\author{
HE Ping ${ }^{1}$, WANG Yingying ${ }^{2}$, YUE Shaohua ${ }^{2}$ \\ (1. Information Engineering Academy, Xijing University, Xi'an 710123, China; \\ 2. Air and Missile Defense College, Air Force Engineering University, Xi'an 710046, China)
}

\begin{abstract}
To compute the operating range of the advanced infrared detector for the stealth aircraft, the combat simulation scenario of F-22 and F-35 is given in the paper. In the scenario, the infrared radiation intensity of F-22 is computed in middle wave-band and long wave-band, the fitting formula of the atmospheric transmittance is given under typical conditions, the characteristic parameters of the F-35 airborne IRST and AIM-9X infrared seeker are inferred and the operating ranges of F-35 airborne IRST and AIM-9X infrared seeker for F-22 are computed with the gradually approaching method. The computation shows that F-22 has good stealth capability and the operating ranges of F-35 airborne IRST is less than 62 $\mathrm{km}$ from the nose of F-22 while the infrared dog-fight missiles threat F-22 in short range strikes. The infrared radiation of F-22 is sensitive to the detection angle of the infrared sensors, thus in the aircraft fleet anti-stealth infrared detection, sparse formation is adopted to increase the detection probability.
\end{abstract}

Key words: stealth aircraft, operating range, infrared search and tracking system(IRST), infrared dog-fight missile, F-22, F-35

\section{0 引言}

随着红外探测技术的发展, 世界上主要的第四代 战机普遍采用红外隐身技术, 以提高战场生存力。比 如 F-22 采用了涂敷红外吸收涂层、二元喷管发动机、 尾翼侧向遮挡等技战术措施, 降低敌方红外传感器发 现、跟踪、识别、攻击的距离和概率 ${ }^{[1]}$ 。
正确估算先进红外传感器对隐身飞机的作用距 离, 是研究隐身飞机红外性能和对其进行探测预警的 基础。红外传感器对隐身飞机作用距离的研究主要涉 及飞机红外辐射、大气红外衰减、红外传感器等方面。 国外研究以美国为代表, 他们将数值仿真与试验测试 手段相结合 ${ }^{[2-5]}$, 并且装备了 F-22、F-117、B-2 等多 种具有红外隐身能力的飞机, 取得了显著成就, 但对 
相关成果严密封锁。国内在飞机红外辐射、大气红外 衰减、红外传感器等方面也做了大量工作 ${ }^{[6-10]}$, 有力 地推动了飞机红外隐身问题的研究, 存在的主要问 题：一是对于飞机红外辐射的计算方法尚无统一规 范, 且采用不同计算方法计算所得的数据差异较大, 导致计算结果互不采纳。二是飞机红外隐身性能并不 能单纯地用红外辐射强度来衡量, 它还与探测飞机所 使用的红外传感器密切相关。F-35 机载的 IRST (infrared search and tracking system) 和 AIM-9X 红外 导引头等红外系统代表了世界红外传感器的领先水 平, 国内对 F-35 的 IRST、AIM-9X 红外导引头等先 进红外传感器基本战技性能研究较多, 而对其传感器 性能参数的深入研究稍显不足。

针对以上问题, 以 F-22 为例, 将 F-22 放在模拟 的战场环境中, 与 F-35 机载的先进红外传感器进行 对抗性仿真研究, 构建 F-22 在长波和中波波段的红 外辐射模型, 拟合典型条件下大气透过率的经验公 式, 并依据公开报道的资料和技术原理, 推测 F-35 机载 IRST 和 AIM-9X 红外导引头的关键参数, 定量 研究典型条件下 F-35 的 IRST 和 AIM-9X 红外导引头 对 F-22 的作用距离, 并利用已掌握的数据, 对研究 结果进行初步校验。

\section{1 红外传感器作用距离模型构建}

将飞机视为点源目标。则红外传感器对飞机的作 用距离 $R$ (单位 $\mathrm{m}$ ) 为 ${ }^{[11]}$ :

$$
R=\left[\frac{\pi \tau_{\mathrm{a}} \tau_{0} D_{0} D^{*}\left(I-I_{\mathrm{b}}\right)}{2 \sqrt{2} F \cdot \mathrm{SNR}}\left(\frac{t_{\mathrm{int}}}{\omega}\right)^{1 / 2}\right]^{1 / 2}
$$

式中: $I$ 为目标的红外辐射强度, $\mathrm{W} / \mathrm{sr} ; I_{\mathrm{b}}$ 为背景的红 外辐射强度, $\mathrm{W} / \mathrm{sr} ; \tau_{0}$ 为光学系统的透过率; $D_{0}$ 为光 学系统入射口径, $m ; F$ 为光学系统的 $F$ 数, $F=f / D_{0}$, $f$ 为光学系统焦距, $m$; $\omega$ 为红外传感器瞬时视场角, $\mathrm{sr} ; D^{*}$ 为在红外传感器响应波长范围内的平均探测率, $\mathrm{m} \cdot \mathrm{W}^{-1} \cdot \mathrm{Hz}^{-0.5} ; t_{\mathrm{int}}$ 为探测器积分时间, $\mathrm{s} ; \mathrm{SNR}$ 为系统 可分辨的极限信噪比; $\tau_{\mathrm{a}}$ 为在红外传感器响应波长范 围内的平均大气透过率。

$\tau_{\mathrm{a}}$ 可表示为:

$$
\tau_{\mathrm{a}}=k \mathrm{e}^{-\gamma R}
$$

式中: $k 、 \gamma$ 为拟合系数。

对于式(1), 等式两边取平方, 得到:

$$
\begin{aligned}
R^{2} & =\frac{\pi \tau_{0} D_{0} D^{*}}{2 \sqrt{2} \mathrm{~F} \cdot \mathrm{SNR}}\left(\frac{t_{\text {int }}}{\omega}\right)^{1 / 2} k \mathrm{e}^{-\gamma R}\left(I-I_{\mathrm{b}}\right) \\
\text { 令 } C & =\frac{k \pi \tau_{0} D_{0} D^{*}}{2 \sqrt{2} \mathrm{~F} \cdot \mathrm{SNR}}\left(\frac{t_{\text {int }}}{\omega}\right)^{1 / 2}, C \text { 为红外传感器综合 }
\end{aligned}
$$

参数; 令 $I-I_{\mathrm{b}}=\Delta I$, 则:

$$
R=[\ln C+\ln (\Delta I)-2 \ln R] / \gamma
$$

由式(4)可知, 红外传感器的作用距离主要与目标 及背景的红外辐射特性、大气的红外辐射传输特性、 红外传感器自身的性能有关。

采用逼近法求 $R$ 值。计算流程如图 1 所示。首先 给定作用距离的期望值 $R_{\mathrm{I} 0}$, 由 $R=[\ln C+\ln (\Delta I)-$ $\left.2 \ln R_{\mathrm{I} 0}\right] / \gamma$ 计算得到的 $R$ 值与 $R_{\mathrm{I} 0}$ 比较, 如果在要求的误 差范围内, 就得到所需的解, 否则修改 $R_{\mathrm{I} 0}$ 值, 直至 $R$ 值在要求的误差范围内为止。

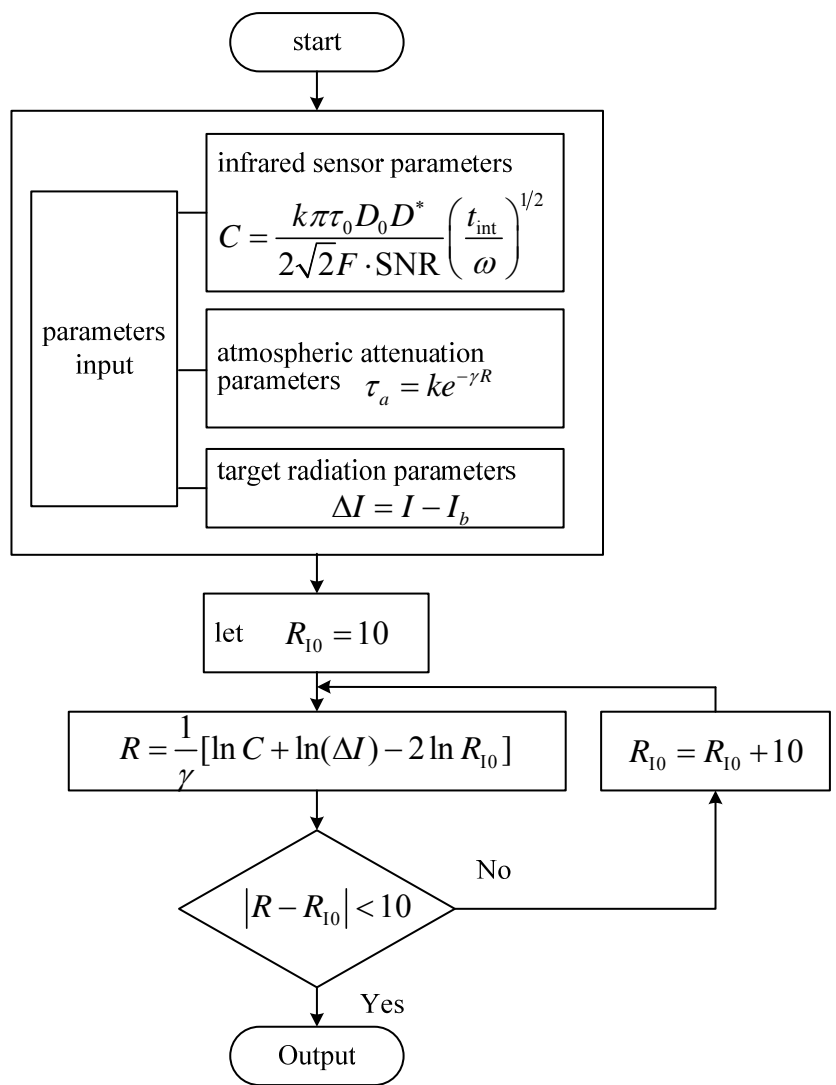

图 1 逼近法求 $R$ 流程图

Fig.1 Flowchart of computing $R$ with the gradually approaching method

\section{F-22 红外辐射计算}

对于涡喷发动机, 综合浴轮叶片材料的热极限和 强度极限, 进入浴轮的气体温度最大值限制在 $1173 \mathrm{~K}$ 左右。就目前的发动机而言, 离开浴轮时的废气温度 EGT (exhaust gas temperature) 高达 $973 \mathrm{~K}$, 只能短时 间内存在, 比如起飞时存在, 在飞机长时间飞行中, 能经受的 $\mathrm{EGT}$ 最大值为 $773 \sim 883 \mathrm{~K}$ 。

根据哈得逊经验公式, 尾喷口外排出的废气温度, 即发动机尾焰温度 $T_{\mathrm{f}}$ 为:

$$
T_{\mathrm{f}} \approx 0.85 \mathrm{EGT}
$$

相比浴喷发动机, 隐身飞机采用的浴扇发动机尾 
喷管、尾焰的温度要低些。仿真计算中, 假设 F-22 浴扇发动机尾喷管可能的温度范围为 $508 \sim 828 \mathrm{~K}$, 则 对应尾焰可能的温度范围为 $432 \sim 704 \mathrm{~K}^{[12-13]}$ 。

已知 F-22 机长 $18.92 \mathrm{~m}$, 机高 $5.08 \mathrm{~m}$, 翼展 $13.56 \mathrm{~m}$, 机翼展弦比 $2.4^{[1]}$ 。估算得到飞机特征面积为 $140.1 \mathrm{~m}^{2}$, 侧视面积为 $45.6 \mathrm{~m}^{2}$, 前/后视面积为 $23.3 \mathrm{~m}^{2}$ 。假定蒙皮 光谱发射率可能的取值范围为 $0.1 \sim 0.5$ 。发动机尾喷管 后视面积为 $1.08 \mathrm{~m}^{2}$, 尾喷管顶/仰视面积为 $1.3 \mathrm{~m}^{2}$, 尾 喷管光谱发射率取 0.9 。尾焰顶/仰/侧视面积为 $1 \mathrm{~m}^{2}$, 后视面积为 $0.2 \mathrm{~m}^{2}$, 尾焰光谱发射率取 0.5 。假设 F-22 飞行马赫数为 $0.9 \sim 2.0$ 。

采用普朗克公式可求出 F-22 在给定波段内各辐 射源的红外辐射亮度, 根据各辐射源在不同方向上的 辐射面积, 可计算出各辐射源的红外辐射强度。为了 得到飞机红外辐射强度方向图, 设在机翼平面上, 探 测点和机体的连线与机体纵轴间的夹角为 $\alpha$ 。则飞机 在 $0^{\circ} \sim 360^{\circ}$ 方位上红外辐射强度 $I_{1}$ 为:

$$
I_{1}= \begin{cases}I_{\text {后视 }} \cdot \cos \alpha+I_{\text {侧视 }} \cdot \sin \alpha & 0 \leq \alpha \leq 0.5 \pi \\ I_{\text {前视 }} \cdot \cos (\pi-\alpha)+I_{\text {侧视 }} \cdot \sin \alpha & 0.5 \pi<\alpha \leq \pi \\ I_{\text {前视 }} \cdot \cos (\alpha-\pi)+I_{\text {侧视 }} \cdot \sin (\alpha-\pi) & \pi<\alpha \leq 1.5 \pi \\ I_{\text {后视 }} \cdot \cos \alpha+I_{\text {侧视 }} \cdot \sin (\alpha-\pi) & 1.5 \pi<\alpha \leq 2 \pi\end{cases}
$$

通过仿真计算比较, 当 F-22 飞行马赫数为 1.6 , 蒙皮发射率为 0.1 , 发动机尾喷管温度为 $588 \mathrm{~K}$, 尾焰 温度为 $500 \mathrm{~K}$ 时, 在采用红外吸波涂料技术、二元喷 管技术、遮挡技术等综合隐身措施后, 可使前半球 8 $12 \mu \mathrm{m}$ 波段红外特征降低约 90\%, 下降比例如图 2 所 示, 与文献[14-15]报道的 F-22 的红外隐身能力基本 吻合。

因此, 在下面计算中, F-22 蒙皮发射率取 0.1, 浴扇发动机尾喷管温度取 $588 \mathrm{~K}$, 尾焰温度取 $500 \mathrm{~K}$ 。

长波探测时, 当 $\mathrm{F}-22$ 马赫数为 $0.9 \sim 2.0$ 时计算 得到 $0^{\circ} \sim 360^{\circ}$ 方位上的红外辐射强度如图 3 所示。中 波探测时, 当 F-22 飞行马赫数为 0.9 和 2.0 , 计算得 到 $0^{\circ} \sim 360^{\circ}$ 方位上的红外辐射强度如图 4 所示。

由图 3、图 4 可知:

1）长波探测时, F-22 飞行马赫数越大, 飞机的 红外辐射强度越大; 不同的探测角度下飞机的红外辐 射差异较大; 由于发动机的贡献, F-22 后半球红外辐 射强度大于前半球。

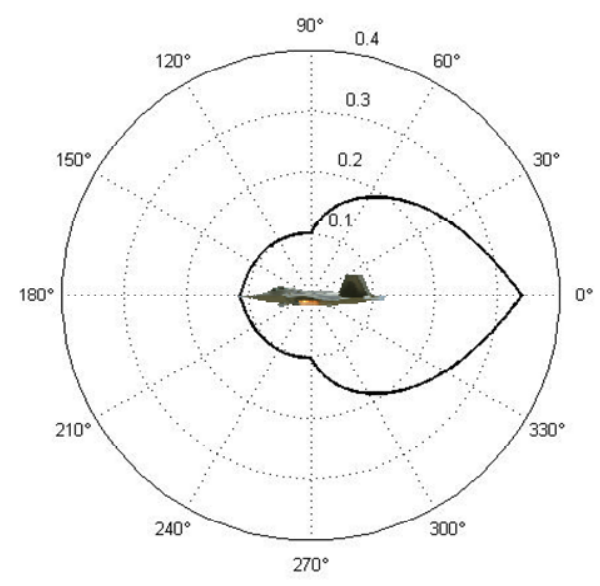

图 2 F-22 在 8 12 $\mu \mathrm{m}$ 波段红外辐射下降比例图

Fig.2 Infrared radiation reduction ratio in 8-12 $\mu \mathrm{m}$ wave-band

2) 中波探测时，由于发动机尾喷管、尾焰为主 要的中波红外辐射源, 蒙皮辐射与之相比、较为微弱, 因此 F-22 后半球红外辐射强度远大于前半球。

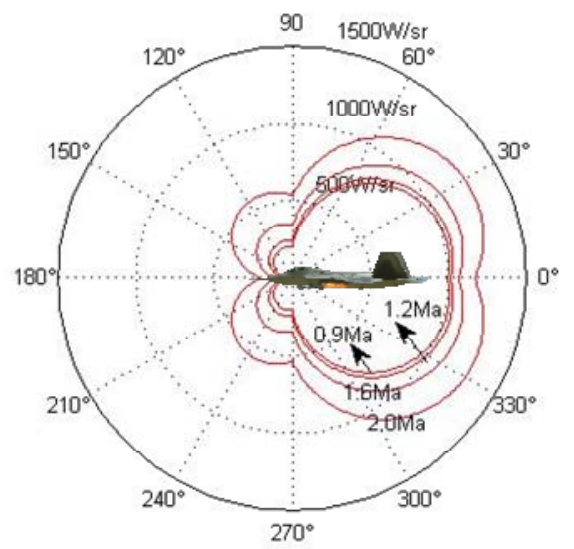

图 3 F-22 在 $8 \sim 12 \mu \mathrm{m}$ 波段红外辐射方向图

Fig.3 Infrared radiation pattern of F-22 in 8-12 $\mu \mathrm{m}$ wave-band

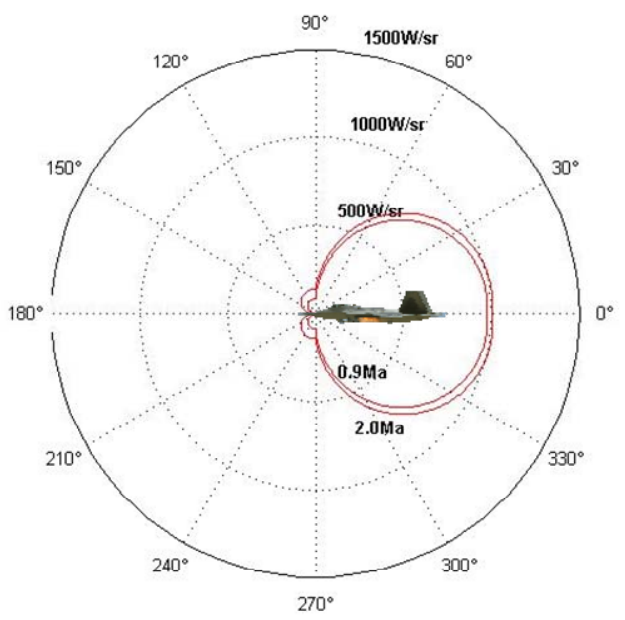

图 4 F-22 在 3 $5 \mu \mathrm{m}$ 波段红外辐射方向图

Fig.4 Infrared radiation pattern of F-22 in 3-5 $\mu \mathrm{m}$ wave-band 


\section{3 大气透过率计算}

依据作战想定研究, 利用 F-35 机载 IRST 来探测 F-22, 可假定 F-22 飞行高度为 $12 \mathrm{~km}$; 利用 F-35 机载 AIM-9X 与 F-22 进行对抗时, 可假定 F-22 飞行高度 为 $5 \mathrm{~km}$ 。由于 F-35 机载 IRST 工作波段为 $8 \sim 12 \mu \mathrm{m}$, AIM-9X 工作波段为 3 5 $\mu \mathrm{m}$, 因此, 对飞机飞行高 度为 $5 \mathrm{~km}$ 时中波段、 $12 \mathrm{~km}$ 时长波段的大气透过率曲 线进行拟合。

\section{1 高度 $12 \mathrm{~km}$ 的大气透过率曲线拟合}

大气条件: 中纬度春夏季, 气溶胶模型为乡村消 光系数, 缺省气象视距为 $23 \mathrm{~km}$ 。利用红外大气传输 软件的原始数据, 飞机飞行高度为 $12 \mathrm{~km}, 8 \sim 12 \mu \mathrm{m}$ 波段平均大气透过率拟合公式为:

$$
\tau_{\mathrm{a}}=0.9534 \mathrm{e}^{-0.0019 R}
$$

拟合曲线如图 5 所示, 拟合精度较高, 最大相对 误差为 $2.2 \%$ 。

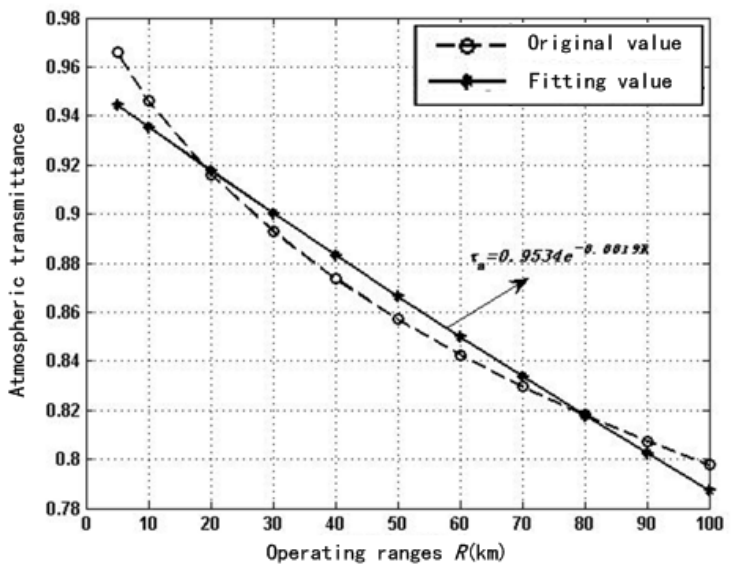

图 5 大气透过率拟合曲线

Fig.5 Atmospheric transmittance fitting curves

\section{2 高度 $5 \mathrm{~km}$ 的大气透过率曲线拟合}

大气条件: 中纬度春夏季, 气溶胶模型为乡村消 光系数、缺省气象视距为 $23 \mathrm{~km}$ 。利用红外大气传输 软件的原始数据, 飞机飞行高度为 $5 \mathrm{~km}, 3 \sim 5 \mu \mathrm{m}$ 波 段平均大气透过率拟合公式为:

$$
\tau_{\mathrm{a}}=0.6954 \mathrm{e}^{-0.0134 R}
$$

拟合曲线如图 6 所示。除个别样本点需剔除之外, 拟合精度较高, 最大相对误差为 $2.28 \%$ 。

\section{4 红外传感器作用距离计算}

\section{1 先进红外传感器参数推测}

\subsubsection{F-35 机载 IRST 参数推测}

采用 $8 \sim 12 \mu \mathrm{m}$ 波段碲镉永探测器, 像元数为
$1 \mathrm{k} \times 1 \mathrm{k}$ 元, 探测器辐射探测率波段平均值为 $7 \times 10^{8}$ $\mathrm{m} \cdot \mathrm{W}^{-1} \cdot \mathrm{Hz}^{-1 / 2}$; 取瞬时视场角 $1^{\circ} \times 1^{\circ}$; 光学系统焦距为 $1.173 \mathrm{~m}$, 取系统 $\mathrm{F}$ 数为 3 , 则系统入射口径为 $0.391 \mathrm{~m}$; 设光学玻璃数为 3 , 则光学系统透过率为 0.86 ; 为了 保证 IRST 系统 $95 \%$ 的探测概率, 信噪比取 5; 积分 时间取 $0.5 \mathrm{~ms}^{[11,16-17]}$ 。

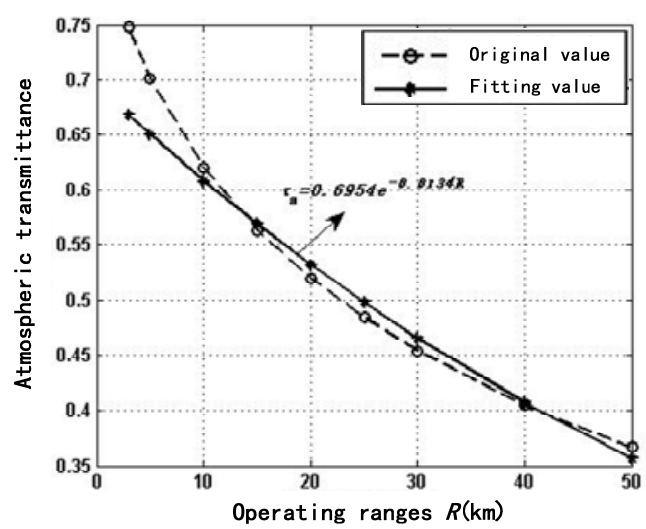

图 6 大气透过率拟合曲线

Fig.6 Atmospheric transmittance fitting curves

\subsubsection{AIM-9X 导引头参数推测}

采用 $3 \sim 5 \mu \mathrm{m}$ 波段碲镉录探测器, 像元数为 $128 \times 128$ 元; 探测器辐射探测率波段平均值取为 $3 \times 10^{9} \mathrm{~m} \cdot \mathrm{W}^{-1} \cdot \mathrm{Hz}^{-1 / 2}$; 导引头瞬时视场角为 $3^{\circ} \times 3^{\circ}$; 光 学系统焦距为 $0.132 \mathrm{~m}$, 取系统 $\mathrm{F}$ 数为 3 , 则系统入射 口径为 $0.044 \mathrm{~m}$; 光学系统透过率为 0.73 ; 信噪比取 5 ;

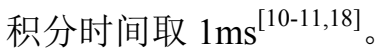

\subsection{F-35 机载 IRST 对 F-22 作用距离计算}

当 F-22 飞行马赫数为 $0.9 \sim 2.0$ 时, F-35 的 IRST 对 F-22 的作用距离如图 7 所示。

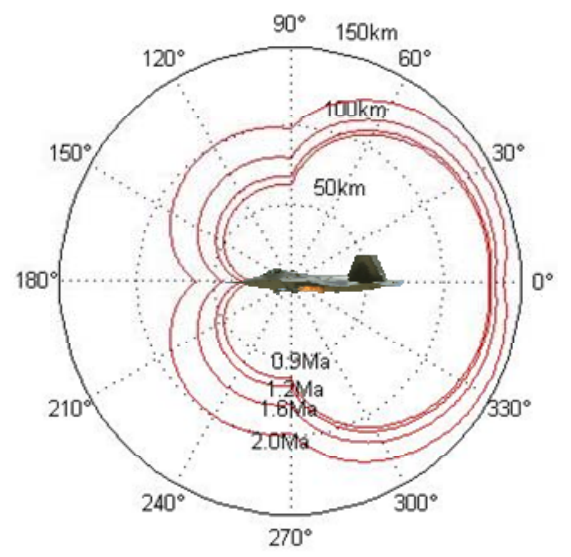

图 7 F-35 机载 IRST 对 F-22 作用距离

Fig.7 Operating ranges of F-35 airborne IRST for F-22

根据图 7 可得到, 随着 F-22 飞行的马赫数不同, 在迎头和尾后两个探测方向上, F-35 的 IRST 对 F-22 的作用距离如表 1 所示。 
表 1 F-35 机载 IRST 对 F22 的作用距离

Table 1 Operating ranges of F-35 airborne IRST for F-22

\begin{tabular}{lccccc}
\hline $\begin{array}{l}\text { F-22 speed } \\
\mathrm{Ma}\end{array}$ & & 2.0 & 1.6 & 1.2 & 0.9 \\
\hline \multirow{2}{*}{$\begin{array}{c}\text { Operating } \\
\text { ranges } / \mathrm{km}\end{array}$} & $\begin{array}{c}\text { nose } \\
\text { direction }\end{array}$ & 61.62 & 44.30 & 31.34 & 24.81 \\
\cline { 2 - 6 } & $\begin{array}{c}\text { tail } \\
\text { direction }\end{array}$ & 138.34 & 132.95 & 130.07 & 129.01 \\
\hline
\end{tabular}

由图 7 和表 1 可知:

1) 当 F-22 飞行马赫数为 $0.9 \sim 2.0$ 时, F-35 的 IRST 对 F-22 迎头的作用距离 $\leqslant 62 \mathrm{~km}$, 据报道, 先进 IRST 对三代机迎头探测距离为 $185 \mathrm{~km}^{[12,14]}$ 。IRST 探测时, 由于迎头探测为主要作战方向, 因此, F-22 的红外隐 身效果显著。

2) F-22 飞行马赫数为 $0.9 \sim 2.0$ 时, F-35 的 IRST 对 F-22 尾后的作用距离 $\geqslant 129 \mathrm{~km}$ 。尾后探测时, 虽然 飞机主要红外辐射源的红外辐射面积比侧视和仰视 时要小, 但由于发动机的贡献, 飞机的红外辐射仍很 大, 因此 F-35 的 IRST 对 F-22 尾后作用距离较大。

3）从 IRST 的角度看, 以 F-22 飞行马赫数为 1.6 为例, 在方位平面上迎头方向 $\pm 30^{\circ}$ 范围内, F-35 机 载 IRST 对 F-22 的作用距离为 $44.3 \sim 69.3 \mathrm{~km}$, IRST 探测角度的变化对传感器作用距离的影响敏感度较 高。因此, 在飞机编队进行红外反隐身作战中, 编队 之间应保持一定的距离差和高度差, 采用稀疏编队方 式, 从而有利于提高编队利用红外传感器发现敌隐身 飞机的概率。

\subsection{AIM-9X 对 F-22 作用距离计算}

AIM-9X 导引头在 3 5 $\mu \mathrm{m}$ 波段探测 F-22 时, 假 设 F-22 飞行马赫数为 0.9 和 2.0 , 计算得到导引头的 作用距离如图 8 所示。

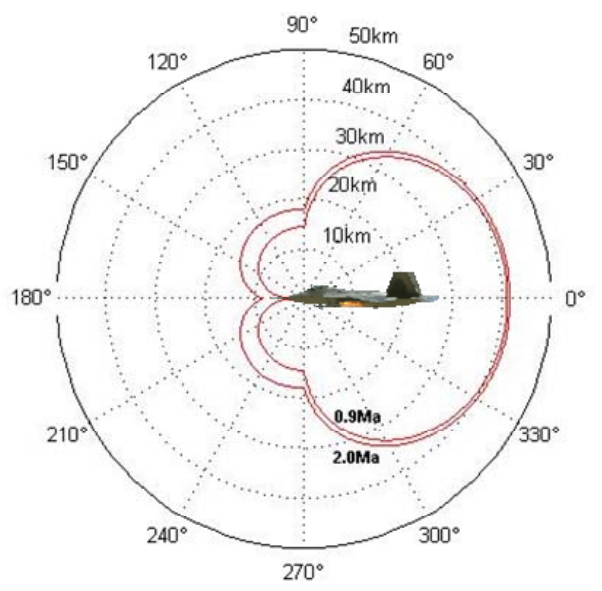

图 8 AIM-9X 导引头的作用距离

Fig.8 Operating ranges of AIM-9X infrared seeker for F-22
由图 8 可知, 当 $\mathrm{F}-22$ 的飞行马赫数为 $0.9 \sim 2.0$ 时, AIM-9X 的导引头对 F-22 前半球作用距离均小于 $20 \mathrm{~km}$, 而后半球的作用距离在 $18 \sim 42 \mathrm{~km}$ 范围内。 由于红外格斗弹攻击时, 尾后攻击为主要作战方向, 因此，F-22 在近距作战中，相比三代机并不具备明显 优势，进入近距作战的 AIM-9X 红外导弹对 F-22 的 威胁较大。

\section{5 结束语}

依据公开资料和技术原理，研究了 F-22 的红外 辐射特性，依据大气红外传输软件，拟合了典型条件 下的大气透过率经验公式, 外推了 F-35 的 IRST 和 AIM-9X 红外导引头的特性参数, 计算了 F-35 的 IRST 和 AIM-9X 红外导引头对 F-22 的作用距离。研究结 果对于飞机红外隐身设计与效能评估，以及红外隐身 反隐身作战方案制订具有一定理论参考。为提升研究 结果的合理性和有效性，可结合高精度飞行器红外特 征软件、大气红外传输软件、红外传感器数据库, 以 及外场它机飞行试验, 对本文研究方法和计算模型进 行校验和修改完善。

\section{参考文献:}

[1] 杨伟. 美国第四代战斗机 F-22“猛禽”[M]. 北京: 航空工业出版社, 2009.

YANG Wei. American Fourth-generation Fighter F-22 Raptor[M] Beijing: Aviation Industry Press, 2009.

[2] 王海晏. 红外辐射及应用[M]. 西安: 西安电子科技大学出版社, 2014. WANG Haiyan. Infrared Radiation and Application[M]. Xi'an: Xi'an University of Electronic Science and Technology Press, 2014.

[3] Gonda T G, Curran A R. Applications of the MuSES infrared signature code[R]. AD-A457152, 2005.

[4] 朱宝錘. 漫谈飞机红外隐身技术 [J]. 兵器知识, 2011(5): 46-47. ZHU Baoliu. Ramble on infrared stealth technology of aircraft[J]. Ordnance Knowledge, 2011(5): 46-47.

[5] 黄臻, 姜伟, 张杨. 飞机红外隐身及探测技术简介[J]. 红外, 2017, 38(8): $1-7$

HUANG Zhen, JIANG Wei, ZHANG Yang. Brief introduction to infrared stealth and detection technology of aircraft[J]. Infrared, 2017, 38(8): 1-7.

[6] 刘娟, 輁光红, 韩亮. 飞机红外辐射特性建模与仿真 [J]. 红外与激光 工程, 2011, 40(7): 1209-1213.

LIU Juan, GONG Guanghong, HAN Liang. Modeling and simulation of airplane infrared characteristic[J]. Infrared and Laser Engineering, 2011, 40(7): 1209-1213.

[7] 李飞. 大气传输对中长波红外辐射衰减分析 [J]. 红外技术, 2019, 41(4): 


\section{1-316.}

LI Fei. Analysis of atmospheric transmission impact on mid-wave and long-wave infrared radiation[J]. Infrared Technology, 2019, 41(4): 311-316.

[8] 何建伟, 曹晨, 张昭. 红外系统对隐身飞机的探测距离分析[J]. 激光 与红外, 2013, 43(11): 1243-1247.

HE Jianwei, CAO Chen, ZHANG Zhao. Analysis on operating range of a IR system for stealth aircraft[J]. Laser \& Infrared, 2013, 43(11): 1243-1247.

[9] 王芳, 罗褱, 王海晏, 等. 机载红外搜索跟踪系统有效探测区域研究 [J]. 激光与红外, 2018, 48(5): 585-590.

WANG Fang, LUO Huan, WANG Haiyan, et al. Research on effective detection area of airborne infrared search and tracking system[J]. Laser \& Infrared, 2018, 48(5): 585-590.

[10] 马晓平, 赵良玉. 红外导引头关键技术国内外研究现状综述 [J]. 航空 兵器, 2018(3): 3-10.

MA Xiaoping, ZHAO Liangyu. An overview of infrared seeker key technologies at home and abroad[J]. Aero Weaponry, 2018(3): 3-10.

[11] RD 小哈得逊. 红外系统原理[M]. 北京: 国防工业出版社, 1975.

Richard D Hudson J R. Infrared System Engineering[M]. Beijing: National Defend Industry Press, 1975.

[12] 牟达, 王建立, 陈涛.凝视型红外搜索跟踪系统对高速飞机作用距离 的分析 [J]. 光学技术, 2007, 32(3): 420-423.

MU Da, WANG Jianli, CHEN Tao. Analysis on operating range of a staring infrared search and track system for high-speed aircraft[J]. Optical Technique, 2007, 32(3): 420-423.
[13] 何苹, 左文博, 杨建军. 预警机红外辐射特性研究[J]. 装备指挥技术 学院学报, 2009, 36(5): 114-116.

HE Ping, ZUO Wenbo, YANG Jianjun. Study on infrared radiation feature of early-warning aircraft[J]. Journal of the Academy of Equipment Command \& Technology, 2009, 36(5): 114-116.

[14] 刘剑. 飞行器红外隐身性能评估系统研究[D]. 南京: 南京理工大学, 2017.

LIU Jian. Infrared Stealth Performance Evaluation System of Aircraft[D]. Nanjing: Nanjing University of Science and Technology, 2017.

[15] 罗明东, 李悦霖. F-22 战斗机红外特征预测模型及飞行测试验证 $[J]$. 国际航空杂志, 2010(11): 60-61.

LUO Mingdong, LI Yuelin. F-22 IR signature flight test model validation[J]. International Aviation, 2010(11): 60-61.

[16] 杨百剑, 万欣. 新一代机载红外搜索跟踪系统技术发展分析 [J]. 激光 与红外, 2011, 41(9): 961-964.

YANG Baijian, WAN Xin. New generation of IRST technology in plane development[J]. Laser \& Infrared, 2011, 41(9): 961-964.

[17] 申洋, 唐明文. 机载红外搜索跟踪系统(IRST)综述[J]. 红外技术, 2003, 25(1): 13-18.

SHEN Yang, TANG Mingwen. An overview of infrared search and track (IRST) system[J]. Infrared Technology, 2003, 25(1): 13-18.

[18] 贾林通, 童中翔, 王超哲, 等. AIM-9X 红外成像制导导弹的发展综述 与启示 [J]. 飞航导弹, 2015(12): 20-24.

JIA Lintong, TONG Zhongxiang, WANG Chaozhe, et al. Development overview and revelations of AIM-9X infra-guided missile[J]. Aerodynamic Missile Journal, 2015(12): 20-24. 